\title{
Evaluating Responsiveness of the Persian Version of the Western Ontario Meniscal Evaluation Tool (WOMET) in Iranian Patients with Meniscal Injury
}

\section{Zahra Jafari Rad}

Iran University of Medical Sciences

Ali Amiri

Iran University of Medical Sciences

Soheil Mansour Sohani ( $\sim$ sohani.soheil@gmail.com )

Iran University of Medical Sciences

\section{Research Article}

Keywords: questionnaire, responsiveness, meniscus, psychometric features

Posted Date: July 6th, 2021

DOl: https://doi.org/10.21203/rs.3.rs-668893/v1

License: (c) (i) This work is licensed under a Creative Commons Attribution 4.0 International License.

Read Full License 


\section{Abstract \\ Background}

This study aimed to evaluate the responsiveness of Western Ontario Meniscal Evaluation Tool (WOMET) and specify its Minimal Clinically Important Difference (MCID) in patients undergoing physiotherapy intervention following undergoing a meniscal lesion or surgery. Our hypothesis was that the WOMET would have adequate responsiveness in patients with meniscal injury.

\section{method:}

100 patients undergoing physiotherapy interventions filled the Persian version of the WOMET and a questionnaire of the Knee injury and Osteoarthritis Outcome Score (KOOS) at Session 1 and Session 10 (4 weeks later). They also filled the 7-point Global Rating of Change (GRC) scale at Session 10. Internal responsiveness was calculated using $T$ test and effect sizes (standard response mean (SRM) and Cohen's d); and external responsiveness was calculated via receiver operating characteristic curve and correlation analysis. The inclusion criterion was the age of 18 to 70 years old for the patients who had the ability of filling the questionnaire. The exclusion criteria included ligaments injury, severe osteoarthritis, inability to complete the questionnaire due to the lack of sufficient knowledge, malignancy, infection, neuromusculoskeletal disorder, rheumatologic disease, knee surgeries for any other reasons, and dissatisfaction for being enrolled in the study.

\section{Results}

All the WOMET subscales (AUC $=0.7$ ) had acceptable external responsiveness. SRM was Trivial, and the scores of Cohen's $d$ were moderate to large and t tests showed significant differences.

\section{Conclusion}

Our findings showed that all of the WOMET subscales had acceptable external responsiveness, and thus this questionnaire could be used to study the effects of physiotherapy interventions on patients undergoing a meniscal lesion or surgery.

\section{Declarations}

\section{Ethics approval and consent to participate}

This research was approved by the local Research Ethics Board (Ethic 
Code:Iran university of medical science,1397.299); Moreover, an informed consent form was taken from all the patients.also all methods were performed in accordance with relevant guidelines and regulations.

\section{Consent for publication}

I have been sent form in separate file

\section{Availability of data and materials}

All data generated or analysed during this study are included in this published article(and its supplementary information files)

\section{Competing interests}

The authors declare that they have no competing interests

\section{Funding}

Iran university of medical science

\section{Authors contributions}

Authors contributions

Choose a topic and idea:all of the authors

Data collection:Zahra Jafari rad

Analysis: all of the authors

Writing and discussion: all of the authors

\section{Acknowledgements}

Not appliable

\section{Introduction}

The meniscus is a biotic structure with different functions, such as load transmission, shock absorption, and stabilization and proprioception. Meniscus injury is a prevalent source of pain associated with functional impairment and articular cartilage damage in long term. ${ }^{1}$ Meniscus injuries can lead to serious health problems and economic burdens due to their high complications and significant impacts on Health-Related Qualities of Life (HRQoL). ${ }^{2}$

HRQoL questionnaires were used to assess patients' general statuses and suggested a way to measure the effects of different therapies. ${ }^{3}$ An effective outcome measure should have three important clinical 
features including validity, reliability, and responsiveness. ${ }^{4-6}$

Being developed by Kirkley et al. ${ }^{7}$ in 2007, the Western Ontario Meniscal Evaluation Tool (WOMET) stands for the first self-reported questionnaire to assess the HRQoL of patients with meniscal pathologies. It consists of 16 questions encompassing three domains of sports/recreation/work/lifestyle (4 items), physical symptoms (9 items), and emotional symptoms (3 items). ${ }^{7-9}$

The reliability and validity of the Persian version of the WOMET have been already evaluated in a group of patients with meniscal lesions ${ }^{10}$; however, its responsiveness and Minimal Clinically Important Difference (MCID) have not been assessed so far.

As a necessary psychometric measurement, responsiveness shows the ability of a tool in delineating clinically significant changes to a patient's health conditions over time. ${ }^{11}$ Internal and external responsiveness statistics are considered as the 2 major aspects determining the changeability of a tool over time and the change limits of an outcome measure proportionate to the changes in the reference standard measures of health conditions, respectively. ${ }^{5,12}$

MCID is defined as the responsiveness of an outcome measure straightly relevant to the amounts of changes in health status, which patients realize. ${ }^{13}$ The purpose of this research was to evaluate the internal and external responsiveness and specify the MCID for the WOMET and the correlations between the WOMET and KOOS questionnaire in patients undergoing physiotherapy interventions after having a meniscal lesion or surgery.

\section{Materials And Methods}

\section{Patients}

100 native Persian-speaking patients undergoing a meniscal lesion or surgery were selected from several physiotherapy centers and orthopedic surgeons' clinics in Tehran, Iran and entered into this study during a period of 12 months (August 2018-2019). The orthopedic surgeons based on the examinations and imaging findings visited all the patients. They must have the age between 18 and 70 years old, and also have the ability to fill the questionnaire to be included in the research. The exclusion criteria were ligaments injury, severe osteoarthritis, and inability to complete the questionnaire for a lack of knowledge, malignancy, infection, neuromusculoskeletal disorder, rhomatological disease, any other knee surgeries, and dissatisfaction for being enrolled in the study.

This research was approved by the local Research Ethics Board (Ethic Code: IR.IUMS.REC.1397.299); Moreover, an informed consent form was taken from all the patients.

\section{Procedure}


The participants at the baseline (Session 1) and 4 weeks later (Session 10) completed the Persian versions of the KOOS questionnaire and WOMET. As a reference standard showing the amounts of changes from the patients' viewpoints, they further completed the 7-point Global Rating of Change (GRC) scale at Session 10. In addition, electrotherapy and specific exercises were incorporated into the physiotherapy interventions.

\section{Outcome measures}

The WOMET consisted of 16 questions representing the three domains of sports/recreation/work/lifestyle (4 items), physical symptoms (nine items), and emotional symptoms (3 items). ${ }^{7-9}$ The scores could be reported totally (0-1600) or separately for each subscale in percentage. ${ }^{14}$

The KOOS questionnaire consisted of 42 items with the five subscales of pain, symptoms, Activity of Daily Living (ADL) scale, sports and recreation (Sports/Rec), and HRQoL. Each item was scored from zero to four. ${ }^{15}$

In most research on responsiveness, the GRC serves as a reference standard, well-validated tool displaying the following seven levels of changes: so much worse=one; much worse=two; slightly worse=three; no change=four; slightly better=five; much better=six; so much better=seven. Based on this scale, the patients were divided into the 2 improved and unimproved groups of so much better and much better levels and slightly better, no-change, slightly worse, much worse and so much worse levels, respectively. $4,16,17$

\section{Statistical analysis}

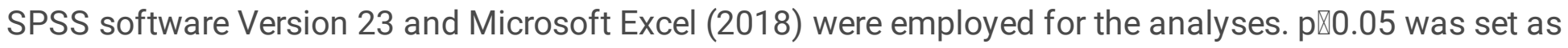
the statistically significant level. The post-intervention scores were subtracted from the pre-intervention scores to calculate the amounts of changes for each questionnaire. ${ }^{18}$ In this investigation, the external responsiveness was examined via the correlation analyses and Receiver Operating Characteristic (ROC) curves; the internal responsiveness was examined using T test effect sizes (SRM and Cohen's d). In the correlation analysis, WOMET questionnaires change scores were correlated with the smallest detectable change (SDC). In addition, we used SDC instead of GRC, because patients may report wrong results. SDC is 20.12 for WOMET questionnaire. Patients who had WOMET scores higher than 20.12 were considered as improved, and who had WOMET scores less than 20.12 were considered as unimproved. For this purpose, the Pearson correlation coefficient was utilized. The correlation coefficients could range from little or no relationship (<0.25), fair (0.25-0.5), moderate to good (0.5-0.75), good to excellent $(\otimes 0.75) .{ }^{19}$ The ROC curve has the two $X$ and $Y$-axis, which are named 1-specificity and sensitivity, respectively. Specificity indicates the ratio of the unimproved patients diagnosed by a tool to their total number diagnosed by the external criteria to be known as truly unimproved. In contrast, sensitivity demonstrates the ratio of the improved patients diagnosed by a tool to their total number diagnosed by the same criteria to be known as truly improved. $., 12,19$ 
The Area under the ROC Curve (AUC) can be interpreted as the probability of an improved patient correctly identified from the selected pairs of the improved and unimproved ones. ${ }^{5,20}$ Showing responsiveness of a tool, this area theoretically ranges from zero (no accuracy) to 1.0 (perfect accuracy). The AUC of greater than 0.70 would be indicative of acceptably high external responsiveness. ${ }^{5,21,22}$ The MCID, which is portrayed near the left corner at top of the diagram, serves as the best cutoff point in the ROC curve and represents the largest specificity and sensitivity. ${ }^{6,19,23}$ It is defined as the number of scores needed to indicate a clinically significant change that is capable of dissociating the improved and unimproved patients. ${ }^{19}$

In this study, we used to test, Cohen's d, and standard response mean for calculating internal responsiveness. Standardized effect sizes of lesser than 0.20 is trivial, 0.20-0.50 and 0.50-0.80 are regarded as the "smallest" and "moderate" sizes, and those above 0.80 can be considered as "large" effect sizes, respectively. The related statistics can be calculated by the following formula:

Where $D$ is the score of raw changes in the measure; SD stands for the standard deviation at time 1; SD* represents the standard deviation of D; ES demonstrates the effect size statistics; D/SD denotes Cohen's d; and D/SD* indicates the Standard Response Mean (SRM). ${ }^{24}$

We used Pair $t$ test to evaluate changes before and after treatment, and independent sample $T$ test was used to assess the changes between groups.

In this study, Pearson correlation between the WOMET and KOOS scales was also calculated. Representing an effect size, correlation strength could be verbally described through Evans' guiding suggestions (1996) for the absolute value of $r:^{25}$

$0.00-0.19=$ very weak

$0.20-0.39=$ weak

0.40-0.59=moderate

$0.60-0.79=$ strong

$0.80-1.0=$ very strong

\section{Results}

Table 1 presents the patients' demographic and clinical features. Table 2 depicts the descriptive statistics for the WOMET subscales and total WOMET scores. 20 and 80 out of 100 patients were categorized as the improved and unimproved ones, in terms of the SDC scale, respectively. Table 3 demonstrates that the WOMET subscales and total WOMET have highly acceptable responsiveness with an AUC of 0.7 and represents the MCID of 20.031 (specificity=1 and sensitivity=1) for the Persian WOMET. 
SRM was trivial for total WOMET, as well as its subscales ( 0.13 for physical symptoms, 0.09 for sport/recreation/work/lifestyle, 0.02 for emotions, and 0.11 for total WOMET); however, Cohen's $d$ was large for total WOMET (-1.586), sports/recreation/work/lifestyle (-1.331), and physical symptoms subscales

(-1.666), and was moderate for WOMET emotions $(-0.705)$ as shown in Table4.

Pearson correlation coefficients were moderate to good for the total WOMET subscales: score $=0.698$, sports/recreation/work/lifestyle $=0.657$ and physical symptoms $=0.723$; however, they were weak (Pearson=0.329) for the emotional subscales as displayed in Table 5. The ROC curves for WOMET questionnaire and its subscales presented in Figure 1 to 4 .

T test showed significant difference before, and after treatments, also between groups as shown in table 6 and 7.

Pearson correlation between WOMET questionnaire and KOOS subscales are shown in Table 8. Regarding, this correlation was ranged from weak to excellent. Overall, there are moderate to high correlation between WOMET and KOOS questionnaires (except emotions subscale).

\section{Discussion}

This study aimed to assess Persian WOMET responsiveness and determining MCID scores in the Iranian patients undergoing meniscal injury. The results revealed that the Persian version of the WOMET has acceptably high external responsiveness as indicated by the AUC values. In addition, moderate-to-good correlations were found between the subscales of sports/recreation/work/lifestyle, physical symptoms, and total WOMET scores and the patients' SDC scales and a weak correlation between the subscale of emotion and SDC scale. Additionally, the MCID of 20.031 points indicated the essentiality of the minimal change score of 20.031 points based on the Persian WOMET for ensuring the occurrence of a true change after physiotherapy interventions. ${ }^{26}$

SRM is trivial; however, the results of Cohen's d showed moderate to large internal responsiveness. In addition, there are significant differences between groups, before and after treatments. External responsiveness is more valid compared to internal responsiveness; therefore, we can state that this questionnaire is responsive, based on good external responsiveness. Pearson's correlation showed moderate to high correlation between WOMET and KOOS questionnaires (except emotions subscale).

The limitations of this study were as follows: First, the use of GRC scale was challenged by the problem of recall bias as discussed by Norman et al. ${ }^{27}$; second, we could not generalize these findings to other languages ${ }^{26}$ and third responsiveness is a population-specific feature. ${ }^{18,20}$

\section{Conclusion}


The results of external responsiveness showed that the Persian version of the WOMET was adequately responsive in patients with meniscal injury, though some contradictory results still existed for SRM and Cohen's d. According to the SRM results, the WOMET had weak (trivial) internal responsiveness, while it displayed good internal responsiveness based on the results of Cohen's $d$ and T tests. Finally, the MCID of the WOMET helped the physiotherapists to understand whether the patients experienced real changes or not. It can be concluded that this questionnaire can be used to study the effects of physiotherapy interventions in patients undergoing a meniscal lesion and surgery.

\section{References}

1. Laible C, Stein DA, Kiridly DN. Meniscal repair. J Am Acad Orthop Surg. 2013;21(4):204-213.

2. Sgaglione NA, Steadman JR, Shaffer B, Miller MD, Fu FH. Current concepts in meniscus surgery: resection to replacement. Arthroscopy. 2003;19 Suppl 1:161-188.

3. Guyatt GH, Feeny DH, Patrick DL. Measuring health-related quality of life. Ann Intern Med. 1993;118(8):622-629.

4. Cleland JA, Whitman JM, Houser JL, Wainner RS, Childs JD. Psychometric properties of selected tests in patients with lumbar spinal stenosis. Spine J. 2012;12(10):921-931.

5. Husted JA, Cook RJ, Farewell VT, Gladman DD. Methods for assessing responsiveness: a critical review and recommendations. J Clin Epidemiol. 2000;53(5):459-468.

6. Stratford PW, Binkley JM, Riddle DL. Health status measures: strategies and analytic methods for assessing change scores. Phys Ther. 1996;76(10):1109-1123.

7. Kirkley A, Griffin S, Whelan D. The development and validation of a quality of life-measurement tool for patients with meniscal pathology: the Western Ontario Meniscal Evaluation Tool (WOMET). Clin J Sport Med. 2007;17(5):349-356.

8. Sihvonen R, Jarvela T, Aho H, Jarvinen TL. Validation of the Western Ontario Meniscal Evaluation Tool (WOMET) for patients with a degenerative meniscal tear: a meniscal pathology-specific quality-oflife index. J Bone Joint Surg Am. 2012;94(10):e65.

9. Tanner SM, Dainty KN, Marx RG, Kirkley A. Knee-specific quality-of-life instruments: which ones measure symptoms and disabilities most important to patients? Am J Sports Med. 2007;35(9):14501458.

10. ebrahimi N JS, Salsabili N. translation and cultural adaptationt reliability and validity study of the persian version of the western ontariomeniscal evaluation tool questionaire in iranian patients with isolated meniscal tears Tehran University of Medical Sciences

School of Rehabilitation; 2016. 
11. Kirshner B, Guyatt G. A methodological framework for assessing health indices. J Chronic Dis. 1985;38(1):27-36.

12. de Yebenes Prous MJ, Rodriguez Salvanes F, Carmona Ortells L. [Responsiveness of outcome measures]. Reumatol Clin. 2008;4(6):240-247.

13. Guyatt G, Walter S, Norman G. Measuring change over time: assessing the usefulness of evaluative instruments. J Chronic Dis. 1987;40(2):171-178.

14. van der Wal RJP, Heemskerk BTJ, van Arkel ERA, Mokkink LB, Thomassen BJW. Translation and Validation of the Dutch Western Ontario Meniscal Evaluation Tool. J Knee Surg. 2017;30(4):314-322.

15. Salavati M, Mazaheri M, Negahban H, et al. Validation of a Persian-version of Knee injury and Osteoarthritis Outcome Score (KOOS) in Iranians with knee injuries. Osteoarthritis Cartilage. 2008;16(10):1178-1182.

16. H T. Reporting improvement from patient-reported outcome measures. Clin Chiropractic. 2010;13:15-22.

17. Kamper SJ, Maher CG, Mackay G. Global rating of change scales: a review of strengths and weaknesses and considerations for design. J Man Manip Ther. 2009;17(3):163-170.

18. de Vet HC, Bouter LM, Bezemer PD, Beurskens AJ. Reproducibility and responsiveness of evaluative outcome measures. Theoretical considerations illustrated by an empirical example. Int $J$ Technol Assess Health Care. 2001;17(4):479-487.

19. Lehman LA, Velozo CA. Ability to detect change in patient function: responsiveness designs and methods of calculation. $J$ Hand Ther. 2010;23(4):361-370; quiz 371.

20. Revicki DA, Cella D, Hays RD, Sloan JA, Lenderking WR, Aaronson NK. Responsiveness and minimal important differences for patient reported outcomes. Health Qual Life Outcomes. 2006;4:70.

21. Irrgang JJ, Anderson AF, Boland AL, et al. Responsiveness of the International Knee Documentation Committee Subjective Knee Form. Am J Sports Med. 2006;34(10):1567-1573.

22. Lin CW, Moseley AM, Refshauge KM, Bundy AC. The lower extremity functional scale has good clinimetric properties in people with ankle fracture. Phys Ther. 2009;89(6):580-588.

23. Bolton JE. Sensitivity and specificity of outcome measures in patients with neck pain: detecting clinically significant improvement. Spine (Phila Pa 1976). 2004;29(21):2410-2417; discussion 2418.

24. Middel B, van Sonderen E. Statistical significant change versus relevant or important change in (quasi) experimental design: some conceptual and methodological problems in estimating magnitude of intervention-related change in health services research. Int J Integr Care. 2002;2:e15. 
25. Alatou ABaD. Precipitation variability on the massif Forest of Mahouna (North Eastern-Algeria)

from 1986 to 2010. International Journal of Management Sciences and Business Research. 2016;5(3).

26. Negahban H, Mostafaee N, Sohani SM, Hessam M, Tabesh H, Montazeri A. Responsiveness and minimally important differences for selected Persian-version of outcome measures used in patients with patellofemoral pain syndrome. Disabil Rehabil. 2015;37(14):1285-1290.

27. Norman GR, Stratford P, Regehr G. Methodological problems in the retrospective computation of responsiveness to change: the lesson of Cronbach. J Clin Epidemiol. 1997;50(8):869-879.

\section{Tables}

Table 1. Demographic and clinical characteristics of patients completing the questionnaires $(\mathrm{n}=100)$

Descriptive statistics

\begin{tabular}{|l|r|}
\hline \multicolumn{2}{|c|}{ n(\%)Unless stated } \\
\hline Demographic data & 1 \\
\hline Missing & $45.55(14.42)$ \\
\hline Age (year), mean (SD)* & $169.6(9.135)$ \\
\hline Height (cm), mean (SD) & $79.16(14.03)$ \\
\hline Weight (kg), mean (SD) & $27.64(5.311)$ \\
\hline BMI & $38(38.4)$ \\
\hline Sex & \\
\hline Men & $61(61.6)$ \\
\hline \multicolumn{1}{|l|}{} \\
\hline Women & $49(49.5)$ \\
\hline Years of education & $50(50.5)$ \\
\hline 112 & $29(29.3)$ \\
\hline Marital status & $70(70.7)$ \\
\hline Single & \\
\hline Couple & $44(44.4)$ \\
\hline Clinical data & $55(55.6)$ \\
\hline Dominant leg & $0(0.0)$ \\
\hline Right & $25(25.3)$ \\
\hline Left & $36(36.4)$ \\
\hline Both & $38(38.4)$ \\
\hline Side of involved knee & $6.20(4.51)$ \\
\hline Right &
\end{tabular}

SD*: standard deviation.

Table 2. Mean (SD) of pre-intervention, post-intervention and change scores for WOMET questionnaire 


\begin{tabular}{|l|l|l|l|}
\hline $\begin{array}{l}\text { WOMET } \\
\text { Questionnaire }\end{array}$ & Pre- & $\begin{array}{c}\text { Post- } \\
\text { intervention: } \\
\text { Mean(SD) }\end{array}$ & $\begin{array}{c}\text { Change: } \\
\text { Mean(SD) }\end{array}$ \\
\hline Total(n=100) & \multicolumn{1}{|c|}{$\begin{array}{c}\text { Mean(SD) } \\
\text { Mervention: }\end{array}$} & & \\
\hline Improved(n=20) & $24.49(16.27)$ & $60.00(13.02)$ & $35.50(14.14)$ \\
\hline $\begin{array}{l}\text { Not } \\
\text { improved(n=80) }\end{array}$ & $49.76(20.71)$ & $43.51(23.47)$ & {$[-6.24(17.98)]$} \\
\hline
\end{tabular}

Table 3. area under the receiver operating characteristic (AUC) curve, sensitivity and 1specificity for WOMET questionnaire. $(\mathrm{n}=100)$

\begin{tabular}{|c|c|c|c|c|}
\hline Questionnaire & AUC $(95 \%$ CI $)$ & $\begin{array}{c}\text { Optimal } \\
\text { cutoff value }\end{array}$ & $\begin{array}{l}\text { Sensitivity } \\
(95 \% \text { CI })\end{array}$ & $\begin{array}{c}\text { 1-Specificity } \\
(95 \% \text { CI) }\end{array}$ \\
\hline WOMET.ps & 0.984 & 22.944 & 0.95 & 0.05 \\
\hline WOMET.S & 0.952 & 15.625 & 0.9 & 0.11 \\
\hline WOMET.e & 0.848 & 4.5 & 0.75 & 0.25 \\
\hline WOMET.t & 1.000 & 20.031 & 1 & 0 \\
\hline
\end{tabular}

All values were significant at $\mathrm{P}<.001$

Table 4.effect sizes 1,2 for WOMET questionnaire and it's subscales

\begin{tabular}{|l|l|l|}
\hline Questionnaire & Cohen's d & SRM \\
\hline dpWOMET.ps & -1.666 & 0.13 \\
\hline dpWOMET.s & -1.331 & 0.09 \\
\hline dpWOMET.e & -0.705 & 0.02 \\
\hline dpWOMET.t & -1.586 & 0.11 \\
\hline
\end{tabular}

dp, difference percent; Ps, physical symptoms; s, sport/recreation/work/lifestyle; e, emotions; t, total; SRM, standard response mean

Table 5. Pearson Correlation Coefficient for WOMET questionnaire and it's subscales

\begin{tabular}{|l|l|}
\hline Questionnaire & Pearson Correlation Coefficient \\
\hline dpWOMET.ps & 0.723 \\
\hline dpWOMET.s & 0.657 \\
\hline dpWOMET.e & 0.329 \\
\hline dpWOMET.t & 0.698 \\
\hline
\end{tabular}


Table 6. Pair T test exam for WOMET questionnaire and it's subscales

\begin{tabular}{|l|l|l|}
\hline Questionnaire & Pair T test & P value \\
\hline dpWOMET.ps & 1.299 & 0.197 \\
\hline dpWOMET.s & 0.899 & 0.371 \\
\hline dpWOMET.e & -0.544 & 0.588 \\
\hline dpWOMET.t & 0.875 & 0.384 \\
\hline
\end{tabular}

Table 7. Independent Sample T test exam for WOMET questionnaire and it's subscales

\begin{tabular}{|l|l|l|}
\hline Questionnaire & Independent Sample T test & P value \\
\hline dpWOMET.ps & -10.358 & $<0.001$ \\
\hline dpWOMET.s & -8.634 & $<0.001$ \\
\hline dpWOMET.e & -3.448 & $<0.001$ \\
\hline dpWOMET.t & -9.651 & $<0.001$ \\
\hline
\end{tabular}

All values were significant at $\mathrm{P}<0.05$

Table 8. pearson correlation between WOMET and KOOS questionnaires

\begin{tabular}{|l|l|l|l|l|}
\hline & dpWOMET.ps & dpWOMET.s & DpWOMET.e & dpWOMET.t \\
\hline dKS.S & 0.667 & 0.621 & 0.315 & 0.651 \\
\hline dKS.pain & 0.770 & 0.803 & 0.447 & 0.795 \\
\hline dKS.ADL & 0.773 & 0.801 & 0.448 & 0.797 \\
\hline dKS.SR & 0.720 & 0.750 & 0.450 & 0.752 \\
\hline dKS.QOL & 0.492 & 0.554 & 0.373 & 0.542 \\
\hline
\end{tabular}

All values were significant at $\mathrm{P}<.001$

dKS, KOOS difference; s, other symptoms

\section{Figures}




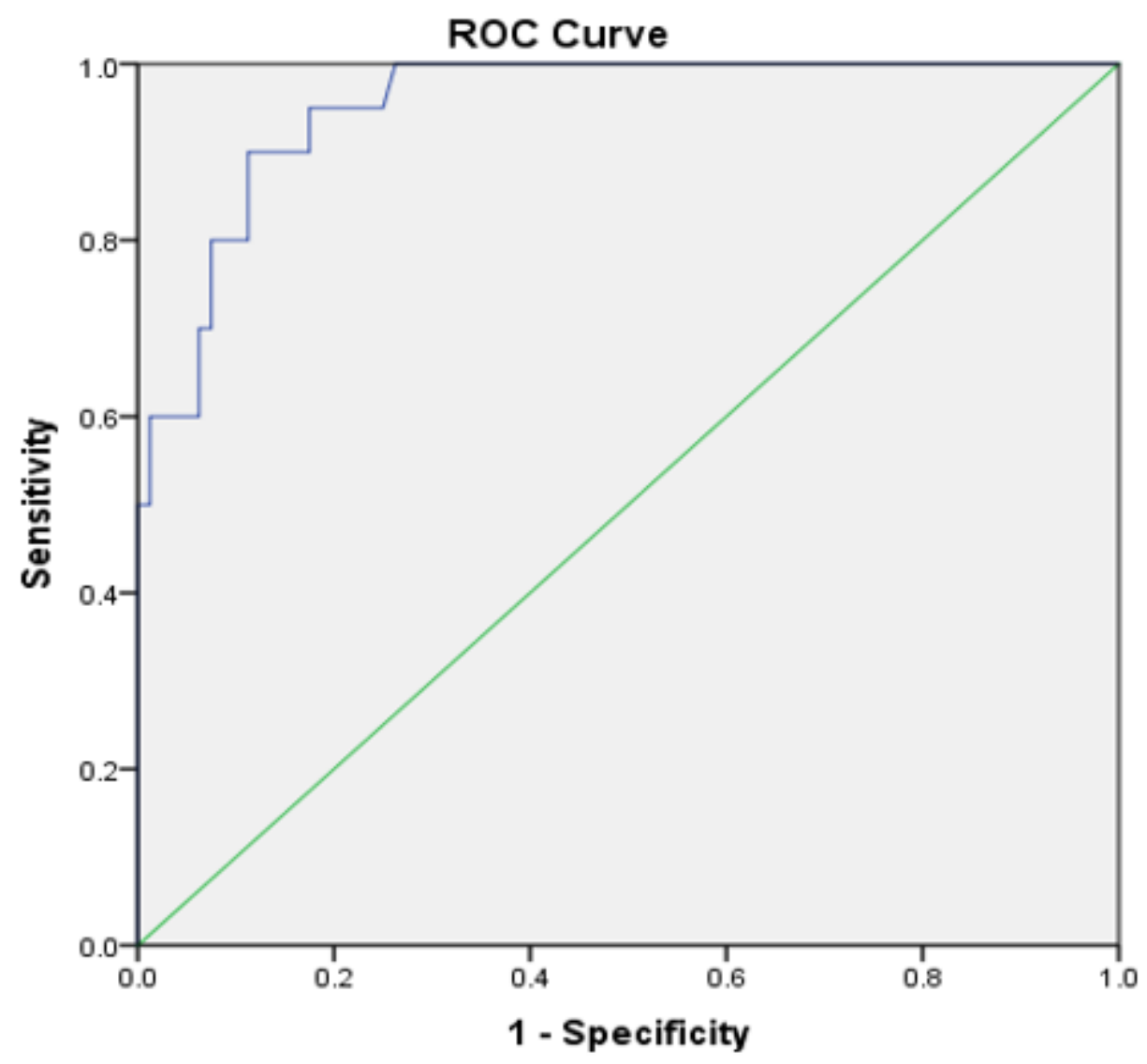

Diagonal segments are produced by ties.

\section{Figure 1}

The receiver operating characteristic (ROC) curves for sport/recreation/work/lifestyle subscale of Persian WOMET questionnaire used in patients with meniscal injury. 


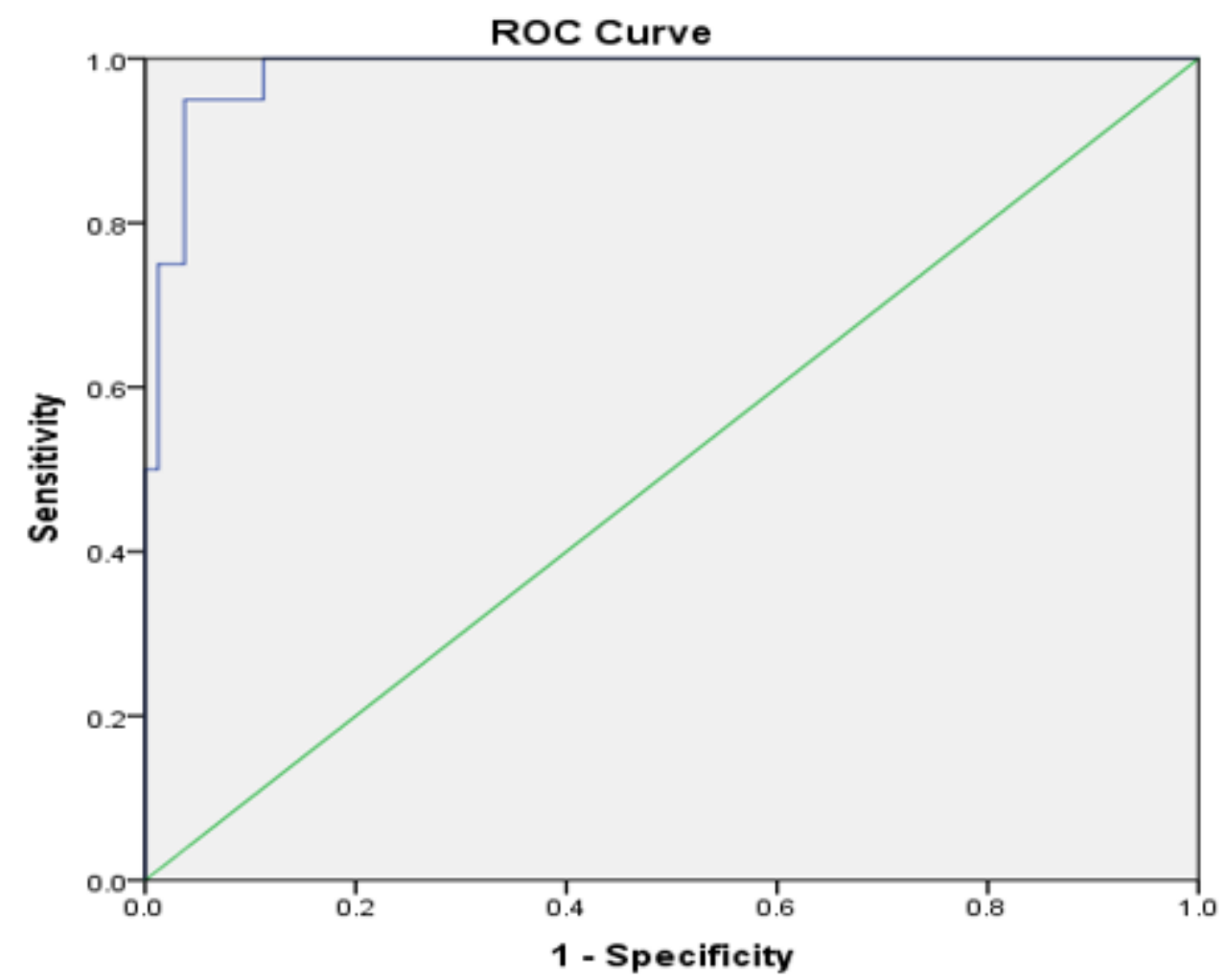

Figure 2

The receiver operating characteristic (ROC) curves for physical symptoms subscale of Persian WOMET questionnaire used in patients with meniscal injury 


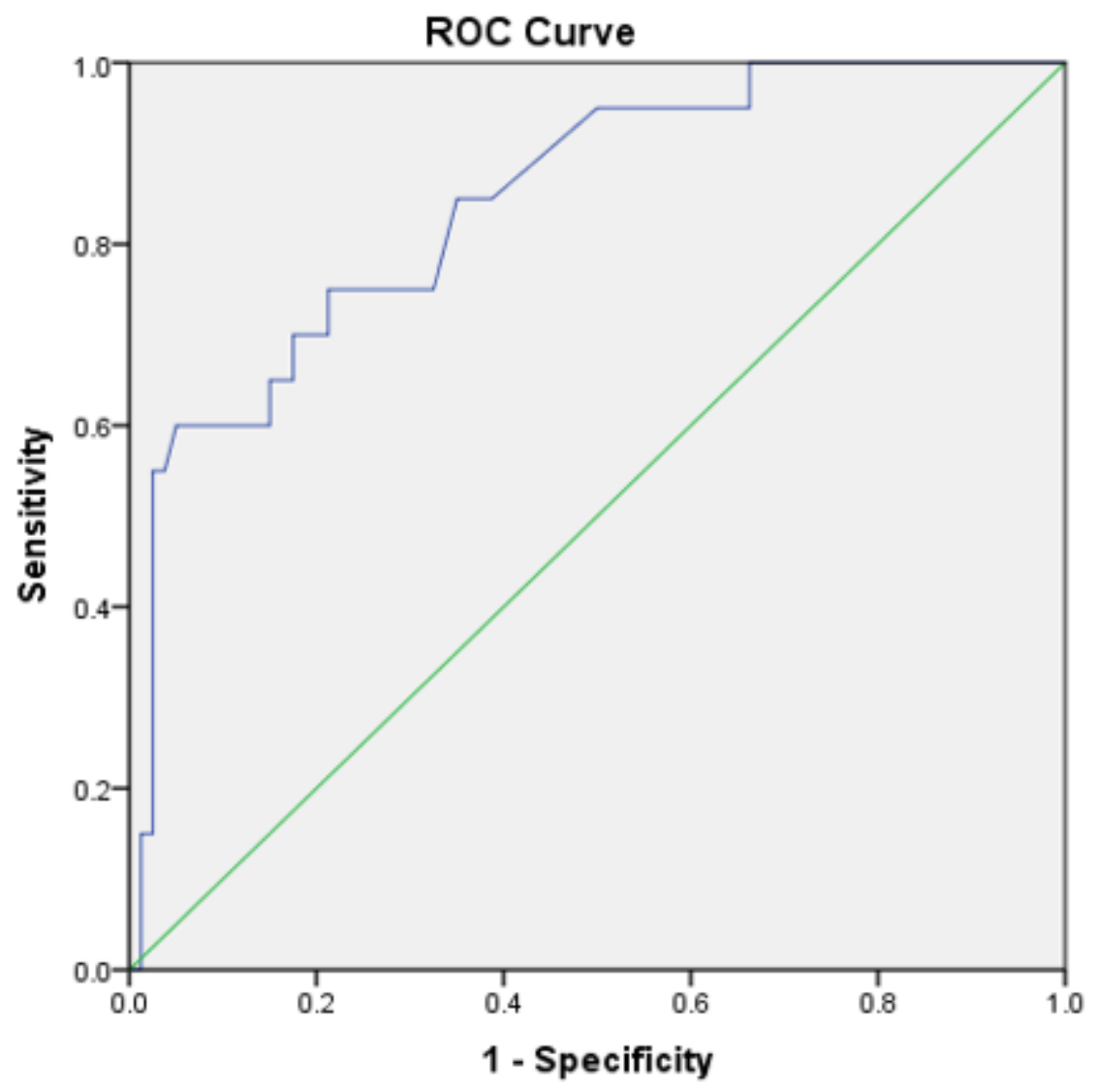

Diagonal segments are produced by ties.

\section{Figure 3}

The receiver operating characteristic (ROC) curves for emotions subscale of Persian WOMET questionnaire used in patients with meniscal injury.

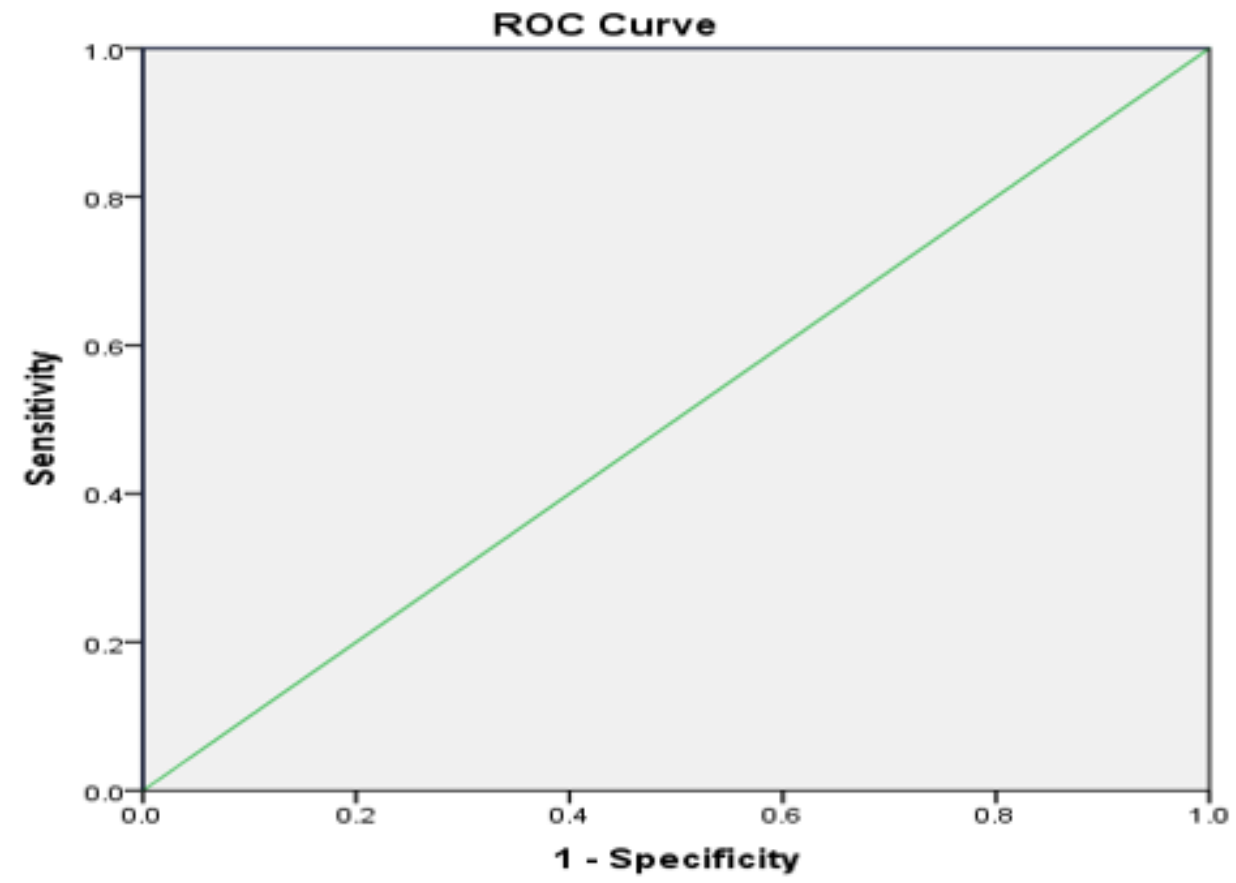

Page 15/16 


\section{Figure 4}

The receiver operating characteristic (ROC) curves for Persian WOMET questionnaire used in patients with meniscal injury. 